\section{A Selective Medium for the Isolation of Soft Rot Coliforms from Soil}

Bacterial species which cause soft rot of plant tissue secrete pectic enzymes which destroy the middle lamella of the cell wall and, in vitro, liquefy pectate gel. This property has been utilized by Wieringa ${ }^{1}$ and Stewart ${ }^{2}$ in the preparation of selective media for isolating soft rotting Erwinia spp., and by Paton ${ }^{3}$ for the isolation of pectin-decomposing Pseudomonas spp. The preparation of these media involves a double-layer technique in which a calcium agar medium is overlaid with a sodium poly. pectate gel in which the formation of shallow pits serves as a diagnostic characteristic.

During investigations of $E$. atroseptica (the causal organism of potato black leg), the selective-diagnostic medium of Stewart ${ }^{2}$, which was developed in these laboratories, has been used for the routine isolation of the organism from diseased plants and tubers and has proved to be extremely efficient for this purpose. On this medium which incorporates MeConkey agar plus calcium chloride in the basal layer, E. atroseptica develops as red colonies in 'cup-shaped' depressions formed in the pectate gel of the upper layer. The bile salts present in McConkey agar inhibit the growth of certain contaminating bacteria.

In examining the survival of $E$. atroseptica in soil where black leg of potato had occurred it was found, by direct soil isolation methods, that as the diseased plant débris rotted there was a marked increase in numbers of saprophytic bacteria, some of which were pectin-decomposing strains of Pseudomonas as described by Paton ${ }^{3}$. On Wieringa's medium these latter types formed depressions indistinguishable from those formed by $E$. atroseptica. On Stewart's medium the fewer colonies of $E$. atroseptica were overgrown and quickly masked by the much more numerous colonies of saprophytic bacteria which included Pseudomonas spp. Replacement of lactose by salicin in the basal layer as suggested by Stewart ${ }^{2}$ was ineffective.

In an attempt to find a more selective substrate the following formula for the basal layer of the medium utilizing sodium citrate as the sole carbon source was devised: Simmon's citrate agar (Oxoid 'C.M.155'), 23.0 g; calcium chloride, $3.0 \mathrm{~g}$; bile salts (Oxoid ' $L .55$ '), $5.0 \mathrm{~g}$; crystal violet, $0.001 \mathrm{~g}$; distilled water, $1 \mathrm{l}$. These ingredients were autoclaved at $15 \mathrm{lb}$. pressure for $15 \mathrm{~min}$, cooled, adjusted to $p \mathrm{H} 6.8$ and $15 \mathrm{ml}$. amounts poured into sterile Petri dishes. The surface of the medium was dried before the addition of $4 \mathrm{ml}$. amounts of the upper layer of pectate solution prepared as described by Paton ${ }^{4}$ and adjusted to $p \mathrm{H} 7.2$. After drying for $12 \mathrm{~h}$ at $37^{\circ} \mathrm{C}$ the pectate gel set firmly and was ready for inoculation.

After incubation for $48 \mathrm{~h}$ at $27^{\circ} \mathrm{C}, E$. atroseptica develops as pale-blue colonies in 'cup-shaped' depressions, $1 \cdot 0-1.5 \mathrm{~mm}$ in diameter, which contrast clearly with the deep-blue colour of the surrounding medium caused by the production of alkali. Other citrate-utilizing coliform types produce pale-blue colonies but cause no liquefaction of the pectate gel. Pseudomonas spp. develop slowly and appear as yellowish-green colonies; the pectin-decomposing types form 'pin-point' depressions, not more than $0.5 \mathrm{~mm}$ diameter, which are easily distinguished from the deeper and larger 'cup-shaped' depressions formed by Erwinia. The latter may be easily counted by holding the plate over a strong light. Although bile salts and crystal violet are necessary when isolating $E$. atroseptica from soils, they may be omitted in its isolation from soft rotted plant or tuber tissues.

Comparisons of this medium with Stewart's shows that it is more selective for $E$. atroseptica. Whether or not this is due to a preference for citrate rather than lactose as a carbon source or to the suppression of the other competitive bacteria is not yet known. The medium has also proved effective for the isolation of $E$. carotovora and $E$. aroideae from soil.

C. LOGAN

Plant Pathology Division,

Ministry of Agriculture,

Queen's University of Belfast.

${ }^{2}$ Wieringa, K. T., Proc. Fourth Congr. Intern. Microbiol., 4, 482 (1949).

${ }^{2}$ Stewart, D. J., Nature, 195, 1023 (1962).

${ }^{3}$ Paton, A. M., Nature, 181, 61 (1958).

'Paton, A. M., Nature, 183, 1812 (1959).

\section{MISCELLANEOUS}

\section{Periodic (Liesegang) Precipitation of Chemicals}

LIESEGANG's periodic precipitation of inorganic chemicals ${ }^{1}$ has yet to be explained adequately, possibly because experiments in gathering data to describe it have failed to recognize and control numerous complicating effects influencing it. Some of these are illustrated in the work reported here.

Reagent grade chemicals, single- or triple-distilled water (glass still), Difco 'Bacto' agar and a purified product of this ${ }^{2}$ were used for this work. (Ion agar No. 2 and 'BBL' agar for bacteriological media were also used, and gave similar results. A process for de-ionization is described by Crowle $^{2}$.) Periodic precipitation was induced in $1-\mathrm{mm}$ layers of 2 per cent agar on $75 \times 50 \mathrm{~mm}$ microscope slides. When internal reactant was used, it was dissolved in the agar before pouring the latter on to a slide. External reactant was allowed to diffuse from $5 \mathrm{~mm}$ disks of EatonDikeman No. 652 filter paper. (By comparison with depot wells punched in agar, this filter paper had no detectable effect of its own on our results, other than that due to absorption of fluid. It was more convenient to use than such wells.) Reactions were allowed to develop in humidified Petri dishes at $2^{\circ} \mathrm{C}$; this temperature was permitted to vary $\pm 2^{\circ}$ or $\pm 0 \cdot 1^{\circ} \mathrm{C}$, depending on the purpose of the experiment. Results reported below are representative of numerous experiments, which among themselves were highly reproducible.

Fig. 1 is a photograph of the numerous Liesegang bands developed overnight at the more constant temperature between 1 per cent silver nitrate and anion contaminants in 'Bacto' agar. This test repeated in de-ionized 'Bacto' agar' ${ }^{2}$ showed no precipitate, unless appropriate anions purposely were re-added to it. The tendency of the unpurified agar to form Liesegang bands diminished upon prolonged boiling during the process of dissolving it.

Several Liesegang bands will form in de-ionized agar in triple-distilled water between $0 \cdot 1$ per cent silver nitrate external reactant and concentrations of sodium chloride internal reactant 100 -fold less than those required to initiate turbidity, detectable by Klett colorimeter, due to silver chloride in liquid, as opposed to gelled, aqueous solution. So sensitive is the agar gel for this effect that brief and light contact with bare fingers, such as occurs when a slide is handled by the edges for a few seconds needed to move it from one place to another, contaminates it with enough chloride ions to form such bands with silver nitrate in the de-ionized system. A similar effect was obtained by making up the de-ionized agar in singlerather than triple-distilled water.

Liesegang bands develop only at certain reactant ratios. This is illustrated in Fig. 1, which shows that until external reactant has diffused a certain distance characteristic for a particular precipitating system the solid disk of precipitate does not begin to break up into concentric rings. But as Fig. 2 shows, such a disk can be broken into similar rings by temperature fluctuations such as occur in the cycling of an ordinary refrigerator (for example, $\pm 0.5^{\circ} \mathrm{C}$ in $10 \mathrm{~min}$ ). Fortunately, by the time the critical reactant ratio favouring true Liesegang precipitation has been reached (for example, immediately outside the solid disk of precipita. 